\title{
[Commentary] Evidence-based policies benefit the men and women who smoke
}

\author{
Gizelle Baker $^{1}$, Jana Olson ${ }^{1}$, Moira Gilchrist ${ }^{1}$ \\ 1 Philip Morris International
}

Funding: The authors are employees of Philip Morris International.

Potential competing interests: The author(s) declared that no potential competing interests exist.

\section{Abstract}

The WHO study group on tobacco product regulation (TobReg) published their eighth report on the scientific basis of tobacco product regulation in May, 2021. The report makes recommendations aimed at addressing "a number of issues concerning novel and emerging nicotine and tobacco products, such as electronic nicotine delivery systems (ENDS), electronic non-nicotine delivery systems (ENNDS) and heated tobacco products (HTPS)". The report concludes by making a number of policy recommendations on HTPS and ENDS that, if implemented, would severely restrict both the availability of these products, and accurate information about them for those adults would otherwise continue to use cigarettes. Whilst the report aims to "protect the health of [...] populations, especially the younger generation", it neglects to consider the potential negative consequences of these recommendations on the more than 1 billion people who smoke. We all agree that smoke-free products carry some risk compared to smoking abstinence or cessation. Nevertheless, the body of available scientific evidence indicates that these products are a better choice for adults who would otherwise continue to smoke.

Rather than promoting regulation that would encourage these adults to switch to better alternatives such as ecigarettes and HTPs, the policy recommendations put forward in the TobReg report will instead maintain the status quo -and support the continuation of smoking, the most harmful form of nicotine and tobacco consumption. The report omits or obscures key information that is vital for science-based policymaking, and there is a clear disconnect between the available body of scientific evidence and the policy recommendations put forth in the report. Here, we share our perspective on the TobReg report in order to contribute to an open, science- and evidence-based dialog.

\section{The 2021 TobReg report recommends continuing the status quo}

The fact that smoking is harmful to health started to emerge in the scientific literature when Doll and Hill published the preliminary results from the British Doctors Study showing a correlation between the smoking habit and many serious diseases, including lung cancer ${ }^{[1][2]}$. Over the decades that followed, new data from this and other studies established the causal link between smoking and a number of smoking-related diseases. This led many countries to implement policies aimed at reducing or eliminating smoking and with it smoking-related harms. In order to pull these disparate, countryspecific approaches into an overarching global strategy, the World Health Organization (WHO) Framework Convention on 
Tobacco Control (FCTC) was adopted in $2003^{[3]}$ to define the tobacco control measures including supply, demand and harm reduction measures intended to reduce smoking rates and improve global population health. Despite the longstanding awareness of the harms of smoking and decades of effort to reduce or eliminate it, today, globally, more than 1 billion people continue to smoke cigarettes. And based on WHO modeling, this number is unlikely to change in the near future ${ }^{[4]}$. Much more can and should be done to expedite progress.

It is recognized that tobacco products exist on a continuum of risk with cigarettes being the most harmfu[p][6]. Consequently, governments and the public health community have repeatedly called on cigarette companies to develop

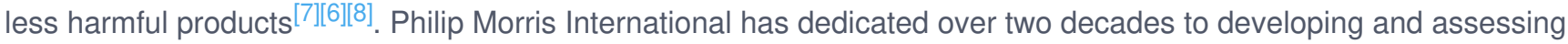
smoke-free products with the potential to reduce the harms and risks of smoking. These smoke-free products do not burn tobacco, and as a result emit significantly lower levels of harmful and potentially harmful chemicals (HPHCs) than cigarettes.

These new products are not risk-free, and they deliver nicotine, which is addictive. For this reason, cessation—completely quitting tobacco and nicotine products all together-is the best choice a smoker can make for their health. Nevertheless, the available scientific evidence clearly supports that smoke-free alternatives are a better choice for adults than continuing to smoke cigarettes. Today however, the WHO has yet to leverage the language in the FCTC to encourage the development of products that have the potential to reduce the harms caused by smoking. Nor have they established policies that enable and encourage adult smokers who do not quit to instead switch to smoke-free alternatives. Ensuring that adult smokers have access to accurate and non-misleading information about these products can help them to differentiate between cigarettes, the most harmful nicotine delivery product, and better alternatives.

It is from this perspective that we reviewed the recent WHO study group on tobacco product regulation (TobReg) report. We recognize that the topic of tobacco harm reduction can be polarizing. Some stakeholders are embracing the role that smoke-free alternatives can play in reducing smoking-related harms, whilst others advocate for application of the precautionary principle and are seeking to effectively ban them. In this atmosphere, it is crucial that the TobReg Report provides a comprehensive and balanced scientific review of all the available evidence to facilitate "scientifically sound, evidence-based recommendations for Member States about tobacco product regulation"[9]. Instead, we found the report to be disconcerting. Key pieces of scientific evidence have been omitted, resulting in conclusions that are misleading, and some of the scientific evidence has been exaggerated in a way that misrepresents the original content and context of the findings. In fact, in some places in the report, undue emphasis is placed on minor findings in order to discredit and undermine some of the key conclusions. Overall, there is a clear and worrying disconnect between scientific evidence and conclusions and recommended policy—something that is at odds with the purpose of the report. It appears as if the science-focused chapters have been framed in order to support a predefined set of conclusions and policy recommendations that, if implemented, would stifle the progress being made both in developing less toxic alternatives to cigarettes and in moving adults who would otherwise continue to smoke, away from cigarettes.

Whilst this response is not a comprehensive point-by-point analysis of the report-and we do not suggest that we alone have the answers on these important topics—we are sharing our perspective, based on our scientific research and 
understanding, as well as our knowledge of adult smokers, in order to engage in a dialogue and broader discussion on these areas of research and analysis.

\section{Shifting stance on the role of smoke-free products in population harm reduction}

In the past, TobReg has accepted less toxic alternatives as a potentially important part in reducing the harm caused by tobacco, and that regulation should encourage their use over more harmful products. In 2015 they wrote: "Development of new tobacco products that are less toxic or less addictive could be a component of a comprehensive approach to reducing tobacco-related deaths and disease, particularly among tobacco users who are unwilling to quit or are unable to break their dependence on tobacco." [10] They also added, "Comprehensive regulation should encourage the use of products that are less toxic, such as medicinal nicotine and nicotine delivery devices, and should reduce the availability and appeal of more toxic products." ${ }^{[10]}$

The policy recommendations in the 2021 TobReg report stands in stark contrast to these earlier statements. Despite the expanding scientific evidence base supporting harm reduction in the intervening years, the report now recommends bans and significant limitations on smoke-free products that would make it difficult, or impossible, for adult smokers to gain access to products that are a better choice than continuing to smoke cigarettes.

For example, the report proposes to ban open tank e-cigarettes, despite ASH UK's evidence that over $70 \%$ of e-cigarette users in Great Britain are using open tank systems ${ }^{[11]}$. Whilst open tank systems do pose a challenge for regulators, an outright ban would mean the loss of one of today's most impactful alternatives to cigarettes in that market. Additionally, the report recommends applying the strictest regulation to all tobacco products equally. This would effectively prohibit communicating information about innovations such as heated tobacco products. The unintended consequence of this policy recommendation will be to favor existing combustible products, such as cigarettes. Preventing current adult smokers from learning that there are alternatives, and why switching to these less toxic alternatives is a better choice than continuing to smoke cigarettes, will deprive them of crucial information that could help motivate these adults to move down the continuum of risk.

The recommendations in the 2021 TobReg report—if implemented as written-would prohibit risk-related communications, even when such information is substantiated by peer-reviewed scientific data from the manufacturer. This approach is the antithesis of the approach taken for other regulated products in the market, including the approach taken for tobacco and nicotine products by one of the world's leading regulators, the U.S. Food and Drug Administration (FDA). TobReg's current policy proposals would ultimately deny adult smokers truthful accurate information about the products that are available to them today. Preventing smokers from being able to make informed decisions for their own health will ultimately be detrimental to public health overall.

\section{The best policies consider all the available evidence}

Policy decisions should be informed by the entirety of the available scientific evidence base and should include rational analysis. Indeed, research from WHO itself shows that evidence-based policies and practices produce better outcomes $^{[12]}$. Avoiding inappropriate influence of vested interests in policymaking is clearly important, but it does not 
mean that interested and impacted parties should not be engaged in the discussion. Instead, their involvement should be regulated and monitored to avoid undue influence. In most, if not all, regulated sectors (e.g., pharmaceutical products and medical devices) the industry is engaged on key issues affecting them and their products. When dealing with pharmaceutical products and policies, the WHO involves manufacturers, and the data that they generate on their products throughout the process. In contrast, when dealing with tobacco products, the 2021 TobReg report minimizes the contribution of industry science and expertise, and their data are either outright ignored or misrepresented in a way that it no longer accurately reflects the context of the research or meaning of the data.

For example, our own extensive scientific research into IQOS (PMI's HTP) shows that there are fewer and on average significantly lower levels of HPHCs emitted in the IQOS aerosol than cigarette smoke. A number of independent studies and regulatory studies or reviews have confirmed this. However, the TobReg report states that: "IQOS aerosol may contain other chemical constituents not present in tobacco smoke, which have not yet been investigated in untargeted analyses" (emphasis added). This concept (that IQOS aerosol is not fully explored or understood) is reiterated in several places throughout the report and yet it is patently false and misleading. In fact, nearly $100 \%$ of the mass of IQOS aerosol has been characterized in our untargeted aerosol chemistry analysis which is published in the scientific literature ${ }^{[13]}$. This research was cited in at least one chapter of the 2021 TobReg report, while being completely ignored in others.

There are ways to engage with the industry-especially on products and science-that can avoid inappropriate influence, but also permit a comprehensive scientific debate that would not only result in informed policy, but also drive the industry to develop better products and science.

\section{The manufacturer's role in the science of tobacco harm reduction}

One of the more striking inconsistencies in the 2021 TobReg report is the disconnect between their recommendation that the burden of proof for product claims lies with the manufacturer, while at the same time recommending that the manufacturer's evidence is insufficient to authorize claims. We agree with the authors of the TobReg report that the product-specific burden of proof lies with the manufacturer. This is normal practice in all other industries and implies that a manufacturer's research must be reviewed and considered. When considering whether a new product can reduce a smoker's exposure to toxicants or reduce a smoker's risk of smoking related disease, all of the available evidence on the product should be subject to scrutiny by regulatory bodies, just as was done in the U.S. by the FDA. Products simply cannot be scientifically substantiated prior to commercialization without the companies commissioning and funding studies. It would be extraordinary to expect that burden of substantiation to be placed on non-commercial entities. If TobReg's policy recommendation were implemented, it would simply lead to an impasse: no one would invest to develop the science that can be used to provide truthful information to guide adult smoker's choices.

Although the initial data—and a sizable proportion of the current evidence base—comes from commercial entities, there is a growing body of independent evidence on the risks and potential benefits of novel and emerging tobacco and nicotine products. This is exactly what can be expected for any new product in any sector. Manufacturers are obliged to assess their products before commercializing them and independent researchers cannot study a new product until it is 
commercially available. However, over the last 5-10 years more and more products have entered the market and as a result the body of independent evidence is growing rapidly. Today, much more is known about chemical emissions, product toxicity, and even reductions in exposure to harmful chemicals in smokers who completely switch to these products from cigarettes. We acknowledge that long-term studies on disease outcomes are still several years away, however, that does not mean that there is insufficient evidence to act now. As an example, although the results do not imply a causal relationship, in Japan we see an encouraging declining trend in the hospital admission rate for both ischemic heart disease and chronic obstructive pulmonary disease (COPD) exacerbations that temporally coincide with the introduction of heated tobacco products like IQOS. And it is consistent with a large number of smokers moving away from cigarettes and what is observed in studies of short-term indictors following cessation or switching to smoke-free alternatives. Instead of seizing the opportunity that today's body of evidence brings from a public health perspective, the TobReg report chooses to focus on what is not known—and cannot be known today—as a reason for recommending policies that will inevitably inhibit progress for adult smokers.

Regulators, health agencies, and tobacco control advocates from around the world have long held the opinion that manufacturers should develop better products. These opinions were not based on ideology or vested interests; they were grounded in scientific evidence, acceptance of the principles of harm reduction and with the right degree of focus on adults who smoke. It has taken decades to develop such products, with several commercial failures along the road, but today industry has developed, scientifically assessed, and is marketing products for adult smokers that are patently better than continuing to smoke cigarettes. Now that this has been achieved, the goalposts are actively being moved.

Stakeholders who once thought many lives could be saved by implementing regulations that permit a manufacturer who produces a genuinely less hazardous product, and develops sound scientific evidence of its impact, to responsibly make claims about any such innovative product ${ }^{[14]}$, now suggest that this is not good enough. Today, they would rather double down on the decades-old strategies of prevention and cessation in an attempt to somehow maintain the status quo.

\section{Assess and communicate risks relative to continued smoking}

There is scientific consensus that smoke-free products do carry some risk compared to smoking abstinence, or cessation, and it is important that adult smokers and the general public are informed of this. We believe that these products must be regulated and marketed responsibly to adults who would otherwise continue to smoke cigarettes. Nevertheless, evidence shows that today there are globally more than one billion smokers and that in any given year, less than 1 in every 10 of them will successfully quit smoking cigarettes ${ }^{[15]}$. In our view, ignoring the opportunity that smoke-free products can bring to these people is not an option, despite the fact that they are not risk-free. If these alternatives are not made available to adult smokers, or if they are not motivated to switch to them-the most likely outcome is that many of them will continue to smoke cigarettes.

Therefore, whilst it is important that smoke-free products are available to adult smokers, it is also imperative that they receive accurate information about them relative to continuing to smoke. In order to motivate adults to switch away from cigarettes, they must (1) be made aware of the fact that better alternatives exist, (2) know what these products are and how they are different from cigarettes, (3) understand who these products are for (and just as importantly who they are not 
for), and (4) be informed about and understand both the risks and the benefits of switching completely and abandoning cigarettes.

Communicating risk is a complex challenge. It requires a balance between the individual's right to know scientific and technical information that is central to them making an informed decision, and the understandability of the risk messages[xvii]. The challenge is to convey the message in a way that unintended audiences (e.g., youth and nonsmokers and others who should not use smoke-free products) will understand that these products are not for them, whilst at the same time, conveying the important role that these products play for adults who would otherwise continue to smoke cigarettes, so that they understand that completely replacing cigarettes with smoke-free products is far better than continuing to smoke.

Everyone communicating about tobacco harm reduction products has a responsibility to present the risks and benefits accurately and in the right context to each relevant audience. When communicating to non-smokers, describing the absolute risks is important, whereas when communicating with adult smokers, a comparison with both the risk of on-going smoking and smoking cessation becomes important. For this audience, it is also important that they understand that there is an absolute risk of using these products. However, communications focused solely on this would make it impossible for them to understand the impact that switching can have as opposed to continuing to smoke. Worse still, incomplete information or context can leave adults who smoke guessing as to whether smoke-free products are a better or worse choice than continuing to smoke cigarettes.

Whilst these absolute and relative risk concepts are important in consumer communications, they are just as important in scientific studies. The lack of an appropriate comparator can lead to study conclusion that are out of context and are ultimately misleading. The 2021 TobReg report includes several studies that either do not have a comparator or the wrong comparator (they compare the results of smoke-free products exposure to fresh air instead of cigarette smoke exposure). These results do not provide the reader with vital context to help in the evaluation of results and their communication to other researchers, public health professionals, policymakers, the public, and—most importantly—adults who smoke.

\section{New products require new and standardized analysis methods}

The authors of the 2021 TobReg report argue that tobacco research would benefit from a harmonization of research methods. We agree. When methods are harmonized or similar across studies, it is easier to compare between products and to determine what the overall evidence shows for a product or category of products. We believe that a collaborative approach is needed to develop a set of standardized methods across the range of tobacco research topics so that everyone can move the research forward and build a fuller understanding of impacts of tobacco harm reduction.

As an example, researchers studying tobacco product use patterns currently lack a common ontology or standard set of definitions for different types of use patterns. Many studies broadly define "dual use" as concomitant use of two different tobacco products, while others consider that cigarettes must be one of the two products-even in broad terms these definitions have very different meanings. When looking at the details of such studies, categorization of people into different product use groups becomes even more challenging. For example, the length of tobacco use surveyed (past 7- 
day use, past 30-day use, ever use), how much of each product is being used, what percent of the tobacco/nicotine consumption is coming from each product, or how many days they are using each of the products all become important.

Differences in the way these factors are measured can lead to a misunderstanding of the results and sometime misleading results that do not accurately reflect real-world use patterns.

Standardization of research methods such as survey questions, product use definitions or categories, sampling population and methods and follow-up intervals (e.g., a snapshot in time vs. follow-up over time) can help build an understanding about what is happening in the real world. Critical questions such as "is dual use a permanent use pattern or a phenomenon related to progression toward complete switching?" The current lack of consistency makes it difficult to accurately compare results between different studies and therefore impossible to gain a full and accurate understanding of the different tobacco product use patterns.

We ourselves recognized the impact of not having a common set of standards and definitions even within our own research program and worked to develop our methods and definitions so they are fit for purpose across multiple study designs and to help ensure that the results from the different studies in our product assessment program can build on one another. A common set of definitions and standards, developed as a collaboration between the industry, independent scientists, and regulators alike, would benefit all parties. These definitions would facilitate quantifiable and comparable measurements that are accepted by all parties to establish facts. This would streamline the review of evidence packages (good for regulators), shorten the review timeline (good for manufacturers), and ensure parity between claims used on different products (good for consumers).

\section{Re-focus tobacco policy on the consumer}

Given the intention of the TobReg report-to help inform regulations, we believe that the underpinning scientific evidence base should be thoroughly reported, debated, and evaluated. We believe that all sources of scientific evidence should be reviewed and considered, given the variety of conflicts of interest that exist.

We recognize that our own interest is primarily commercial and that our priority and focus is to convince adult smokers to switch to better alternatives, whilst being mindful to avoid unintended consequences. The policy recommendations in the TobReg report prioritize concerns of nonsmokers with a focus on protecting this group from using smoke-free products. In doing so, the proposals outlined aim to restrict or prohibit access to these products and limit information about them. Whilst we agree, it is important to minimize the number of non-smokers who initiate with smoke-free products, we argue that there is no inherent conflict between these two objectives outlined above. Both are critical areas to focus on, to maximize the population benefit from smoke-free alternatives. We believe that by working together with all stakeholders, it is perfectly possible to achieve both.

Regulatory measures, such as enforcing a minimum age for sales with strict age verification requirements, controls on the use and marketing of flavors to minimize youth appeal, advertising controls on content and placement of material to ensure that they target adult consumers, bans on misleading and unsubstantiated messages, and product standards that set minimum quality and safety requirements for these products can aid in both objectives. These measures should be 
defined and implemented in a way that is proportionate to the risk of the product, placing the strictest controls on the most risky products—-those that combust tobacco.

Sound science is fundamental to any decision making or policy formation approach. By recommending evidence-based policies that encourage targeted communications with factual information, the WHO and TobReg could continue to support prevention and quitting as the gold standards, and also be instrumental in accelerating the end of cigarettes, by maximizing the number of adult smokers who switch to better alternatives, whilst minimizing the number of nonsmokers who take up these products.

We suggest that instead of polarizing the debate, by all stakeholders working together and being mindful of both objectives, it is perfectly possible to make cigarettes obsolete in many countries in 10-15 years. To achieve this goal, continued enforcement and strengthening of the tobacco control policies for cigarettes and other combustible tobacco products is required. At the same time leveraging the opportunity of smoke-free products-and all the science that supports them-can dramatically accelerate progress. All stakeholders should be advocating together with and on behalf of consumers, to ensure that they receive accurate and non-misleading information about innovative alternatives to cigarettes. Protecting the vulnerable need not come at the expense of the billion people on the planet who smoke today. Through appropriate science-based policy and regulations, let adult consumers see the evidence for themselves and make their own decisions.

\section{References}

1. ^R. Doll, A. B. Hill. (1950). Smoking and Carcinoma of the Lung. BMJ, vol. 2 (4682), 739-748. doi:10.1136/bmj.2.4682.739.

2. 'Maria Elisa Di Cicco, Vincenzo Ragazzo, Tiago Jacinto. (2016). Mortality in relation to smoking: the British Doctors Study. Breathe, vol. 12 (3), 275-276. doi:10.1183/20734735.013416.

3. 'The WHO Framework Convention on Tobacco Control (FCTC). doi:10.1057/9780230361249.0014.

4. ^ (2019). WHO global report on trends in prevalence of tobacco smoking 2000-2025, second edition. Geneva:. World Health Organization.

5. ^ (1989). Improving Risk Communication. doi:10.17226/1189.

6. ${ }^{a}, \mathrm{~b}(2001)$. Clearing the Smoke. doi:10.17226/10029.

7. 'Dietrich Hoffmann, Ilse Hoffmann, Karam El-Bayoumy. (2001). The Less Harmful Cigarette: A Controversial Issue. A Tribute to Ernst L. Wynder. Chem. Res. Toxicol., vol. 14 (7), 767-790. doi:10.1021/tx000260u.

8. ^Royal College of Physicians. (2007). Harm reduction in nicotine addiction: helping people who can't quit. A report by the Tobacco Advisory Group of the Royal College of Physicians. London.

9. ' (2021). WHO study group on tobacco product regulation: Report on the scientific basis of tobacco product regulation: eighth report of a WHO study group. Geneva. World Health Organization.

10. ${ }^{a, b}(2015)$. WHO study group on tobacco product regulation: Report on the scientific basis of tobacco product regulation: eighth report of a WHO study group. Geneva. World Health Organization.

11. ^Action on Smoking and Health. (2021). Use of e-cigarettes (vapes) among adults in Great Britain. 
12. ^(2005). WHO: A Toolkit for Progressive Policymakers in Developing Countries. Geneva. World Health Organization.

13. 'Mark C. Bentley, Martin Almstetter, Daniel Arndt, Arno Knorr, et al. (2020).Comprehensive chemical characterization of the aerosol generated by a heated tobacco product by untargeted screening. Anal Bioanal Chem, vol. 412 (11), 2675-2685. doi:10.1007/s00216-020-02502-1.

14. 'US Senate. (2007). The Need for FDA Regulation of Tobacco" Transcript from the Hearing of the Committee on Health, Education, Labor and Pensions.

15. ' USHHS. (2020). Smoking Cessation: A Report of the Surgeon. US Surgeon General. 